\title{
Barrier option pricing for assets with Markov-modulated dividends
}

\author{
Giuseppe Di Graziano* \\ Statistical Laboratory \\ University of Cambridge \\ L.C.G ROGERS ${ }^{\dagger}$ \\ Statistical Laboratory \\ University of Cambridge
}

19th April 2005

\begin{abstract}
We present a simple methodology to price single and double barrier options when the dividend process of the underlying is a Markov-modulated log-Brownian motion, and the stock is priced in equilibrium by a CRRA representative agent. In particular, we show how to derive the Laplace transform (in time) of the barrier price, by solving a system of ODEs. The method proposed is extremely simple to implement but also extremely effective. Pricing of double barrier option in the classical Black and Scholes framework arises as a special case of the model presented in the paper.
\end{abstract}

Keywords: barrier option, Markov-modulated, dividends, Laplace transform.

\footnotetext{
*Wilberforce Road, Cambridge CB3 0WB, UK (phone $=+441223$ 9798, e-mail = gd259@cam.ac.uk).

${ }^{\dagger}$ Wilberforce Road, Cambridge CB3 0WB, UK (phone $=+441223$ 766806, fax $=+441223337956$, e-mail = L.C.G.Rogers@statslab.cam.ac.uk).
} 


\section{Introduction}

In this paper, we present a simple but fairly general methodology to price a variety of barrier options when the dividend process of the underlying is a Markov-modulated log-Brownian motion, as in Di Graziano and Rogers [DGR]. Assuming a CRRA representative agent and equilibrium pricing leads to the dynamics (2) of the stock; see [DGR] for the derivation, the main conclusions of which are summarised in Section2. The pricing problem reduces to solving a system of second order linear PDEs with appropriate boundary and terminal conditions, which we simplify to a system second order linear ODEs by taking the Laplace transform (with respect to time) of the option price. The ODE system can be solved explicitly and option prices are recovered by numerically inverting the Laplace transform. Sections 3 and 4 explain in detail how this is done.

A similar methodology has been used in Jobert and Rogers [JR]. Their modelling assumptions are different from those here, in that the dynamics of the stock price are directly modelled as a Markov-modulated log-Brownian motion. They use a generalization of the Rogers-Stapleton [RS] binomial method to approximate the price of the barrier option, whereas we obtain (at least for constant barriers) an exact solution for the Laplace transform of the option price which we then invert numerically.

The methodology can also be applied to price (double) barrier options in the classical Black-Scholes framework, which is a special case of the model presented in this paper; in Section 5 we compare the results of doing this with other approaches to this problem from the literature.

The methods presented in this paper can be extended, to include for example different dynamics for the underlying process; the key fact in the approach is that we do not attempt any finite-difference approximation to the diffusion part, but just handle the problem through exact solutions to ODEs, joined together by Markov chain conditions and technology, and this is not limited to the familiar log-Brownian motion.

\section{The model}

Following Di Graziano and Rogers [DGR], we assume that the dividend process $\delta$ solves the SDE

$$
\frac{d \delta_{t}}{\delta_{t}}=\left[\sigma\left(\xi_{t}\right) d W_{t}+\gamma\left(\xi_{t}\right) d t\right]
$$


where $W$ is a standard Brownian motion, $\gamma$ and $\sigma$ denote the Markov-modulated drift and volatility of the process $\delta$ under the pricing measure and $\xi$ is a finite-state irreducible Markov-chain. It is shown in [DGR] that if (1) is interpreted as the dividend process of the single production activity in an economy with a single representative agent with CRRA utility, then equilibrium considerations lead to a stock price process

$$
S_{t}=\delta_{t} v\left(\xi_{t}\right)
$$

where the function $v$ is given by

$$
\begin{gathered}
v=(\rho-Q-F)^{-1} \mathbf{1}, \\
F=\operatorname{diag}(f), \\
f(x) \equiv(1-R)\left(\mu-\frac{1}{2} \sigma^{2}\right)(x)+\frac{1}{2}(1-R)^{2} \sigma^{2}(x),
\end{gathered}
$$

where $\mu$ is the drift of the dividend process under the physical measure, $\rho$ and $R$ are the representative agent's discount coefficient and coefficient of relative risk aversion respectively. The risk-neutral drift $\gamma$ and the drift $\mu$ under the physical measure are related by the following simple expression (see [DGR] for details)

$$
\gamma(\xi)=\mu(\xi)-R \sigma^{2}(\xi)
$$

\section{Barrier Options}

In this section we will show how to price barrier options when the dynamics of the stock process follow $^{1}(2)$. In particular, we will show how to price down-and-in and downand-out put options. Up-and-in and up-and-out call and put prices can be recovered in a similar fashion, modulo some change in the boundary condition of the relevant ODEs. Double-barriers can be also handled using the same methodology, only the coding becomes slightly more complicated.

It turns out that the problem simplifies significantly if we price down-and-in type options and then recover the down-and-out via parity arguments.

A down-and-in put can be thought of as digital option which delivers one unit of a vanilla put option at the random time when the barrier is crossed. In order to price

\footnotetext{
${ }^{1}$ The methodology presented is more general and can in principle be adapted to other underlying diffusion processes.
} 
the down-and-in option then, we will first solve for the (time to maturity) Laplace transform of the vanilla put and then derive an expression for transform of the digital option.

Under the pricing measure $Q$, the value of a vanilla put option is given by

$$
P\left(t, \delta_{t}, \xi_{t}\right)=E_{t}\left[\exp \left(-\int_{t}^{T} r\left(\xi_{u}\right) d u\right)\left(K-\delta_{T} v\left(\xi_{T}\right)\right)^{+}\right],
$$

where $r$ is the Markov-modulated short-rate process. Note that in the set up of Di Graziano and Rogers [DGR] $r$ takes the form

$$
r(x)=\rho+R\left(\mu-\frac{1}{2} \sigma^{2}\right)(x)-\frac{1}{2} R^{2} \sigma^{2}(x) .
$$

Let now $\tau \equiv T-t$ denoted the time to maturity of the option. With a slight abuse of notation we can define the Laplace transform with respect to the time to maturity as

$$
\phi(\delta, \xi)=\int_{0}^{\infty} e^{-\tau p} P(\tau, \delta, \xi) d \tau \text {. }
$$

Routine calculations lead us to the system of ODEs which must be satisfied by (8):

$$
\frac{1}{2} \delta^{2} \sigma^{2} \phi^{\prime \prime}(\delta)+\delta \gamma \phi^{\prime}(\delta)+(Q-r-p I) \phi(\delta)+(K-\delta v)^{+}=0
$$

where $\phi(\delta)$ is a vector-valued function whose $i^{t h}$ component is given by $\phi(\delta, i), Q$ is the infinitesimal generator of the chain, and $\sigma^{2}, \gamma$ and $r$ are diagonal matrices whose $i^{t h}$ diagonal elements are equal to $\sigma^{2}(i), \gamma(i)$ and $r(i)$ respectively.

By letting $y=\log (\delta)$ and defining $\varphi(y)=\phi(\delta)$, the ODE system (9) reduces to

$$
\frac{1}{2} \sigma^{2} \varphi^{\prime \prime}(y)+\left(\gamma-\frac{1}{2} \sigma^{2}\right) \varphi^{\prime}(y)+(Q-r-p I) \varphi(y)+\left(K-e^{y} v\right)^{+}=0 .
$$

The homogenous part of system (10) admits solutions of the form

$$
\varphi(y)=a e^{\lambda y},
$$

where $a$ is a vector of dimension $d$ and $\lambda$ is a scalar. Substituting (11) into (10), after multiplying all the terms in (10) by $2 \sigma^{-2}$, we obtain the following system of equations to be solved in $\lambda$ and $a$,

$$
\lambda^{2} a+\lambda\left(2 \sigma^{-2} \gamma-I\right) a+2 \sigma^{-2}(Q-r-p I) a=0,
$$


which, by letting $h=\lambda a$ can be reformulated as

$$
M\left(\begin{array}{c}
h \\
a
\end{array}\right)=\left(\begin{array}{cc}
I-2 \sigma^{-2} \gamma & 2 \sigma^{-2}(p I+r-Q) \\
I & 0
\end{array}\right)\left(\begin{array}{c}
h \\
a
\end{array}\right)=\lambda\left(\begin{array}{c}
h \\
a
\end{array}\right) .
$$

Now, (13) is a standard eigenvalue problem. Following [BRW], it can be proved that there are exactly $d$ eigenvalues in the left half-plane and $d$ in the right-half plane.

Let now $\bar{K}_{i}=\log \left(K / v_{i}\right)$ and assume, without loss of generality, that the states of the chain were labeled in such a way that $\bar{K}_{1} \leq \bar{K}_{2} \leq \cdots \leq \bar{K}_{d}$. The solution space of (10) can be divided in $d+1$ regions. We consider the solution in the region $\left[0, \bar{K}_{1}\right]$. Since the payoff of a put option is bounded, as $y \downarrow-\infty$ (or equivalently as $\delta \downarrow 0$ ), the put price must tend to a finite limit. As a consequence we must restrict our attention to the positive eigenvalues only of system (13). For $y \leq \bar{K}_{1}$ then, the solution of the homogenous part of (13) can be represented as the weighted sum

$$
\varphi_{0}(y)=\sum_{i=1}^{d} w_{0 i} e^{\lambda_{i} y} a_{i}
$$

where $\operatorname{Re}\left(\lambda_{i}\right)>0$ for $i=1, \ldots, d$ and the coefficients $w_{0 i}$ need to be determined.

In the region under consideration, the inhomogeneous part of (10) simplifies to $K-e^{y} v$, where $K$ is a vector whose entries are all equal to the strike $K$. The particular solution of this system will have the form

$$
\varphi_{P}=g+f e^{y}
$$

Vectors $g$ and $f$ can be derived by substituting (15) back into (10). In particular,

$$
\begin{aligned}
g & =-(Q-r-p I)^{-1} K \\
f & =(\gamma+Q-r-p I)^{-1} v .
\end{aligned}
$$

We have found an expression for the solution of (10) in the interval $\left[0, \bar{K}_{1}\right]$, but we still need to determine the $d$ coefficients $w_{0 i}$.

In order to do so, consider the solution of the ODE system in the intervals $\left[\bar{K}_{s}, \bar{K}_{s+1}\right]$, for $s=1 \ldots d-1$. Since those intervals are all bounded, we can no longer restrict our attention to the positive eigenvalues only of system (13). The solution $\varphi_{s}$ in the intervals $\left[\bar{K}_{s}, \bar{K}_{s+1}\right]$ will then take the form

$$
\varphi_{s}(y)=\sum_{i=1}^{2 d} w_{i s} e^{\lambda_{i} y} a_{i}+g_{s}+e^{y} f_{s} .
$$


where

$$
\begin{aligned}
& g_{s}=-(Q-r-p I)^{-1} K_{s} \\
& f_{s}=(\gamma+Q-r-p I)^{-1} v_{s} .
\end{aligned}
$$

and $K_{s}(j)=K, v_{s}(j)=v_{j}$ for $j>s$ and zero otherwise. Finally in the interval $\left[\bar{K}_{d}, \infty\right)$, we restrict our attention to the $d$ eigenvalues $\lambda_{i}, i=d+1, \ldots, 2 d$ with negative real part. The solution to (10) admits the following representation

$$
\varphi_{d}(y)=\sum_{i=d+1}^{2 d} w_{i d} e^{\lambda_{i} y} a_{i}
$$

In order to fully specify the solution of (10), we need to determine the $2 d^{2}$ unknown coefficients $w_{i s}$. By requiring the solution to be $C^{1}$ at each node $\bar{K}_{s}, s=1, \ldots, d$, for each state $j=1, \ldots, d$, we obtain $2 d^{2}$ linear equations in the $2 d^{2}$ unknowns $w_{i s}$. The specification of the Laplace transform of the put option is thus complete.

\section{Down-and-in put}

Under the pricing measure $Q$, the value of a down-and-in put option is given by

$$
\widetilde{P}\left(t, \delta_{t}, \xi_{t}\right)=E_{t}\left[\exp \left(-\int_{t}^{T} r\left(\xi_{u}\right) d u\right)\left(K-\delta_{T} v\left(\xi_{T}\right)\right)^{+} ; \inf _{t \leq u \leq T} \delta_{u} v\left(\xi_{u}\right) \leq b\right]
$$

where $b$ is the barrier. As mentioned in the previous section, a down-and-in put can be thought of as a digital option which delivers a vanilla put at the time the barrier is crossed.

Define $\bar{b}_{i}=\log \left(b / v_{i}\right)$ and again assume without loss of generality that $\bar{b}_{1} \leq \bar{b}_{2} \leq . . \leq \bar{b}_{d}$. Moreover let

$$
\widetilde{\phi}(\delta, \xi)=\int_{0}^{\infty} e^{-\tau p} \widetilde{P}(\tau, \delta, \xi) d \tau .
$$

Following the line of reasoning of the previous section, it can be easily seen that in the interval $\left[b_{d}, \infty\right), \widetilde{\phi}(\delta)$ solves

$$
\frac{1}{2} \delta^{2} \sigma^{2} \widetilde{\phi}^{\prime \prime}(\delta)+\delta \gamma \widetilde{\phi}^{\prime}(\delta)+(Q-r-p I) \widetilde{\phi}(\delta)=0 .
$$


With a little abuse of notation, it follows that

$$
\widetilde{\varphi}_{d}(y)=\sum_{i=d+1}^{2 d} \widetilde{w}_{i d} e^{\lambda_{i} y} a_{i} .
$$

where the $d$ coefficients $\widetilde{w}_{i d}$ need to be determined. Note that we have restricted our attention to the $d$ eigenvalues with negative real part as the $\varphi_{j d}(y)$ must decrease to zero as $y \rightarrow \infty$.

Suppose now the chain is in state $i=d$ (in our set up the state corresponding to the highest barrier $\left.\bar{b}_{d}\right)$. Once the barrier is crossed, the option becomes a vanilla put; this implies that $\widetilde{\varphi}_{d}(y)=\varphi_{d}(y)$ for $y \leq \bar{b}_{d}$. Continuity at $\bar{b}_{d}$ yields us a first equation for the $d$ unknown weights $\widetilde{w}_{i d}$.

When $y \in\left[\bar{b}_{d-1}, \bar{b}_{d}\right]$, the equation for the down-and-in put changes slightly. In fact, the chain $\xi$ can jump to state $d$, where the down-and-in has the same value as the vanilla put. The function $\tilde{\varphi}_{d-1}$ will thus satisfy the following system of equations

$$
\frac{1}{2} \widehat{\sigma^{2}} \widetilde{\varphi}^{\prime \prime}(y)+\left(\widehat{\gamma}-\frac{1}{2} \widehat{\sigma^{2}}\right) \widetilde{\varphi}^{\prime}(y)+(\widehat{Q}-\widehat{r}-p I) \widetilde{\varphi}(y)+\widehat{q} \varphi_{d}(y)=0
$$

where $\widehat{\sigma^{2}}, \widehat{\gamma}, \widehat{r}$ and $\widehat{Q}$ are obtained from the corresponding $\sigma^{2}, \gamma, r$ and $Q$ by removing the last row and column. The new term $\widehat{q} \varphi_{d}(y)$ is defined so to take into account jumps to state $d$. In particular, $\widehat{q}$ is a $d-1$ dimensional vector such that $\widehat{q}_{i}=Q_{i d}$ for every $i=1, \ldots, d-1$. As before $\varphi_{d}(y)$ is the Laplace transform of the vanilla put for $\xi=d$.

The homogeneous part of equation (26), can be solved similarly to (24). In particular, the eigenvector problem associated to $(26)$ will yield $2(d-1)$ eigenvalues $\widehat{\lambda_{i}}$, with corresponding $2(d-1)$ eigenvectors $\widehat{a}_{i}$. Finding the particular solution is also straightforward but requires some care. As we explained in the previous section, the Laplace transform of the vanilla put is of the form

$$
\varphi_{j}(y)=\sum_{i=1}^{2 d} w_{i} a_{j i} e^{\lambda_{i} y}+g_{j}+h_{j} e^{y} .
$$

with some of the weights $w_{i}$ possibly equal to zero. However, the weights $w_{i}$ as well as vectors $g$ and $h$ depend also on $y$. So if for example, $w_{i}, g$ and $h$ are not constant over the interval $\left[\bar{b}_{d-1}, \bar{b}_{d}\right]$, then we need divide the previous interval in subintervals where the above coefficients are indeed constants and search for a particular solution to (26) in each subinterval. 
For simplicity of exposition, assume that $\bar{b}_{d}<\bar{k}_{1}$, so that all the relevant coefficients in (27) are constant over the interval $\left[\bar{b}_{d-1}, \bar{b}_{d}\right]$. Then the particular solution over this interval will have the form

$$
\widehat{\varphi}_{d-1}^{P}(y)=\sum_{i=1}^{d} c_{i} e^{\widehat{\lambda}_{i} y}+l+e^{y} m,
$$

and the unknown vector coefficients $c, l, m$ can be found the usual way by substituting (28) into (24).

Note that after solving the problem in the interval $\left[\bar{b}_{d-1}, \bar{b}_{d}\right]$ we are left with 1 equation and $d+2(d-1)$ unknowns. By requiring the solution $\varphi_{j}(y)$ to be $C^{1}$ at $\bar{b}_{d}$ for $j=$ $1, \ldots, d-1$ and simply continuous for $j=d$, we obtain $2 d-1$ new equations and we are so left with only $d-2$ degrees of freedom.

We can follow the above procedure backwards up to the interval $\left[\bar{b}_{1}, \bar{b}_{2}\right]$. Finally, for $y \leq \bar{b}_{1}$, the Laplace transform of the down-and-in put coincides with that of the vanilla put for all the states. This completes the specification of the Laplace transform for the down-and-in put.

Remark 1. The price of a down-and-out put can be trivially found as the difference between a vanilla put and a down-and-in put. This comes with no additional computational costs, since we have already derived the Laplace transform of the vanilla put in order to price the down-and-in option.

Mutatis mutandis up-and-in and up-and-out puts can be priced using the methodology described for the corresponding down barriers.

Barrier call prices can be derived following the same methodology after modifying the appropriate boundary and terminal conditions. Indeed, we only need to find the Laplace transform for the vanilla call price. The pricing of the down-and-in call is identical to the one described above (with $\phi(\delta)$ replaced by the transform of the vanilla call)

Remark 2. Pricing double barrier options is also straightforward in the present framework. In particular the price of a double-in put is given by

$$
P\left(t, \delta_{t}, \xi_{t}\right)=E_{t}\left[\exp \left(-\int_{t}^{T} r\left(\xi_{u}\right) d u\right)\left(K-\delta_{T} v\left(\xi_{T}\right)\right)^{+} ; \tau_{l} \wedge \tau_{u}<T\right],
$$

where $\tau_{l}$ and $\tau_{u}$ are the first hitting time of the lower and upper barrier respectively.

In order to derive the price of the double-in put, all we need to do is to simultaneously solve for the Laplace transform of a down-and-in and an up-and-out put using the 
approach highlighted above. More precisely, let $b^{u}, b^{l}$ be the up and down barrier and let $\phi(y)$ be the vector valued Laplace transform of the double barrier (in) put. Then for $y \in\left[\bar{b}_{d}^{l}, \bar{b}_{1}^{u}\right], \varphi(y)$ solves $(24)$, in particular we have that

$$
\varphi_{j}(y)=\sum_{i=1}^{2 d} w_{i} a_{j i} e^{\lambda_{i} y} .
$$

where $\lambda_{i}$ and $a_{i}, i=1 \ldots 2 d$ are the eigenvalues and eigenvectors associated with $(24)$ respectively. On the left of $\bar{b}_{d}^{l}$ we can proceed as in the down-and-in case, whereas on the right of $\bar{b}_{1}^{u}$ we can follow the up-and-in case. As usual we need to determine the unknown coefficients. Imposing the usual continuity conditions at the barriers leaves us with exactly $d$ degree of freedom after solving on the left of $\bar{b}_{d}^{l}$. Proceeding the same way for $y>\bar{b}_{1}^{u}$, we are left with no degree of freedom and the double barrier problem is solved (up to the inversion of the relevant Laplace transform).

Remark 3. If the risk neutral dynamics of stock price process are modeled by a GBM with Markov-modulated coefficients with no jump component of the form (see for example $[\mathrm{JR}])$,

$$
\frac{d S_{t}}{S_{t}}=\left[\sigma\left(\xi_{t}\right) d W_{t}+r\left(\xi_{t}\right) d t\right]
$$

then the approach proposed in this paper simplifies significantly. Both barrier $(\bar{b})$ and strike $(\bar{K})$ thresholds become independent of the state of the chain. This implies that, both for the vanilla put and the down-and-in put, the solution space for the Laplace transform is divided in only two regions. In other words, we need to solve only one eigenvalue problem and $2 d$ linear equations ( $d$ for the vanilla and $d$ for the barrier) to determine the unknown coefficients.

Also, pricing double barrier options amounts to solving $d$ extra equations to determine the $d$ additional unknowns due to presence of the upper barrier. In particular, for $d=1$ (i.e. if we are in the classical Black and Scholes framework), deriving the Laplace transform of the double barrier reduces to solving trivial a second order linear ODE (see $[\mathrm{GY}]$ and $[\mathrm{Pe}]$ for alternative approaches).

\section{$5 \quad$ Numerical examples}

We will now present some numerical examples where we apply the techniques presented in the previous sections. The inversion of the Laplace transform was performed using the Hosono method (see [Ho], [AW]). 
As highlighted by Di Graziano and Rogers ([DGR]), when $\mu(\xi)$ and $\sigma(\xi)$ in (1) are constant functions of the chain $\xi$, we recover the classical Black and Scholes framework for stocks paying a constant dividend rate. For example, for $S_{0}=100, K=100$, $\gamma_{i}=0.05, \sigma_{i}=0.25$, for $i=1 \ldots 2, \rho=0.09$ and $R=3, b=75$ and any $Q$-matrix, the down and in put is worth 11.1442 and the down and out put has a price of 2.9374, which is exactly what we have in the standard Black and Scholes case.

Figure 1 shows the prices of a vanilla, down-and-in and the down-and-out put as a function of the spot, when we use the parameters shown above and $S_{0}$ ranges from 30 to 150 . The barrier is at 60 .

Figure 2 compares prices of standard Black and Scholes down and in puts with barrier at 75, with high volatility $(\sigma=0.35)$ and high drift $(\gamma=0.15)$, low volatility $(\sigma=0.25)$ and low drift $(\gamma=0.1)$, with the prices obtained using the model of [DGR], where we let $\sigma_{1}=0.25, \sigma_{2}=0.35, \gamma_{1}=0.10, \gamma_{2}=0.15, \rho=0.09, R=3$ and $Q$-matrix

$$
Q=\left(\begin{array}{cc}
-1 & 1 \\
3 & -3
\end{array}\right)
$$

As a further check to our methodology, we computed the price of a few double barrier knock-out options. Obviously when coefficients $r$ and $\sigma$ are constant functions of the chain, or equivalently when the chain has only one state, we recover the classical Black and Scholes solution for no dividend paying stocks. We compared our results (see table 5) with those obtained by Geman and Yor [GY], Kunimoto and Ikeda [KI] and Rogers and Zane [RZ2]. In the table below, $L$ stands for lower barrier and $U$ for upper barrier. The table shows that the prices obtained using the method presented in this paper

Table 1: Double barrier knock out prices under different approaches

\begin{tabular}{|c|c|c|c|}
\hline$\sigma$ & 0.2 & 0.5 & 0.5 \\
\hline$r$ & 0.02 & 0.05 & 0.05 \\
\hline$T$ & 1 & 1 & 1 \\
\hline$S_{0}$ & 2 & 2 & 2 \\
\hline$K$ & 2 & 2 & 1.75 \\
\hline$L$ & 1.5 & 1.5 & 1 \\
\hline$U$ & 2.5 & 3 & 3 \\
\hline$G Y$ & 0.0411 & 0.178 & 0.07615 \\
\hline$K I$ & 0.041089 & 0.017856 & 0.076172 \\
\hline$R Z$ & 0.041079 & 0.017837 & 0.076147 \\
\hline$D G R$ & 0.041089 & 0.017857 & 0.076172 \\
\hline
\end{tabular}

match (almost) perfectly those of $[\mathrm{KI}]$ in all the three cases considered. 
Of course, Markov-modulated models are best appreciated when the drift and volatility are non-constant functions of the chain. Assume, for example that $S_{0}=100, K=100$, $U=150$ and $L=75, T=1$ and $\sigma_{1}=0.7071, \sigma_{2}=0.5$ and finally $Q_{12}=0.01$ and $Q_{21}=0.01$, then the price of the double barrier are 1.26 and 3.7930 for initial state 1 and 2 respectively compared with 1.2598 and 3.7932 of [JR].

\section{Conclusions and further research}

We have presented a simple but effective semi-analytical procedure to price various barrier structures when the underlying dividend process is modelled as a log Brownian motion with Markov-modulated coefficients. The procedure simplifies significantly if we model the stock process itself as a log Brownian motion with Markov-modulated coefficient, as in [JR], because of the absence of the jump component. The methodology presented turned out to be accurate, fast and easy to implement. The ideas presented in the paper extend to pricing barrier options with general underlying diffusion dynamics, provided that the corresponding ODE system (9) admits a closed form solution.

\section{References}

[AW] J. Abate and W. Whitt: Numerical inversion of Laplace transforms of probability distributions, ORSA Journal on Computing 7, 36-43, 1995.

[BRW] M.T. Barlow, L.C.G Rogers and D. Williams: Wiener-Hopf factorization for matrices, Seminaire de Probabilites XIV. Lecture Notes in Math, 324-331, 1980. 7, 36-43, 1995.

[CGMY] P. Carr, H. Geman, D. Madan, M. Yor: Stochastic Volatility for Levy Processes, Mathematical Finance, July 2003, Vol. 13, No. 3, 345-382.

[CM] P. Carr and D. Madan: Option pricing and the fast Fourier transform, Journal of Computational Finance 2, 61-73, 1999.

[C] K. Chourdakis: Continous-time regime switching models and application in estimating processes with stochastic volatility and jumps, Working paper, University of London, 2002.

[DGR] G. Di Graziano, L.C.G. Rogers: Equity with Markov-modulated dividends, working paper, University of Cambridge, 2004. 
[GY] H. Geman, M. Yor: Pricing and hedging double barrier options: a probabilistic approach, Mathematical Finance, 1996, Vol. 6, No. 4, 365-378.

[H] S. L. Heston: A closed form solution for options with stochastic volatility with applications to bond and currency options Review of Financial Studies 26, 637-653, 2001.

[Ho] T. Hosono: Numerical inversion of Laplace transform and some applications to wave optics, Radio Science 16, 1015-1019.

[JR] A. Jobert, L.C.G. Rogers: Option pricing with Markov-modulated dynamics, Working paper, University of Cambridge, 2004.

[KI] N. Kunitomo and M. Ikeda: Pricing options with curved boundaries Mathematical Finance, July 1992, Vol. 2, No.4, 275-298.

[KS] I. Karatzas and S. E. Shreve: Methods of Mathematical Finance, SpringerVerlag, New York, 1998.

[KR] R. Korn and L. C. G. Rogers: Stocks paying discrete dividends: modelling and option pricing, Working paper, 2004.

[L] R. W. Lee: Option pricing by transform methods: extensions, unification and error control, Journal of Computational Finance 7, 51-86, 2004.

[Pe] A. Pelsser: Pricing double barrier options using Laplace transform, Finance and Stochastics 7,January 2000, Vol. 4, Iss 1, 95-104.

[Pi] M. Pistorius: American and barrier options under stochastic volatility and jumps, Working Paper, King's College London, 2004.

[P] P. Protter: Stochastic Integration and Differential Equations, SpringerVerlag, New York, 1990.

[RS] L.C.G. Rogers and E.J. Stapleton: Fast accurate binomial pricing. Finance and Stochastics 9 3-17, 1997.

[RW] L.C.G. Rogers and D. Williams: Diffusions, Markov Processes and Martingales: Volume 2 , Ito's Calculus, Cambridge University Press, Cambridge, 2000.

[RZ] L.C.G. Rogers and O. Zane: Saddle-point approximations to option prices, Annals of Applied Probability 9, 493-503, 1999. 
[RZ2] L.C.G. Rogers and O. Zane: Valuing moving barrier options, Journal of Computational Finance 9, 5-11, 1997.

[SST] W. Schoutens, E. Simons, J. Tistaert: A perfect calibration! Now what?, Technical Reports, K. U. Leuven, 2003. 


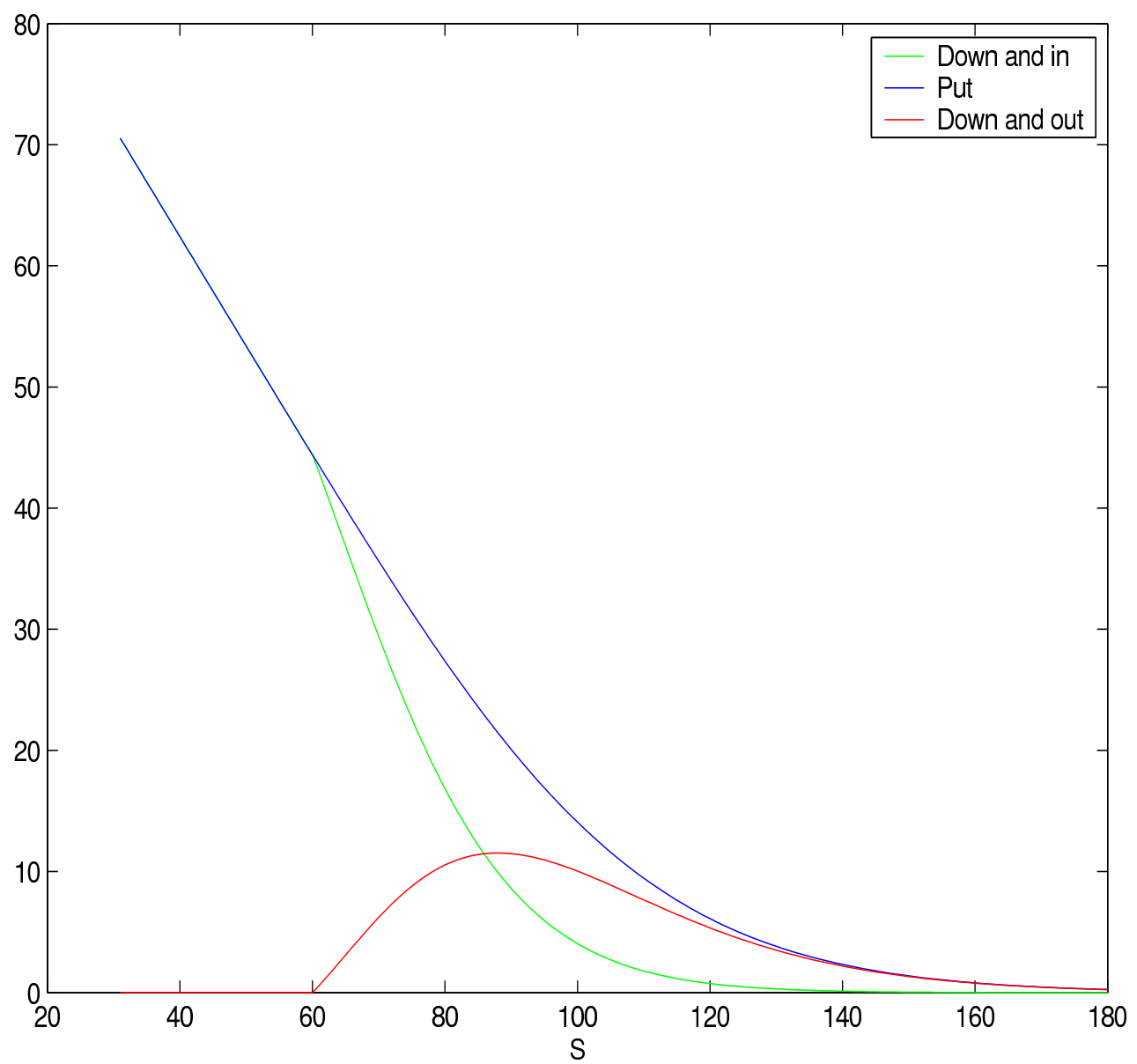

Figure 1: Put prices as functions of spot. 


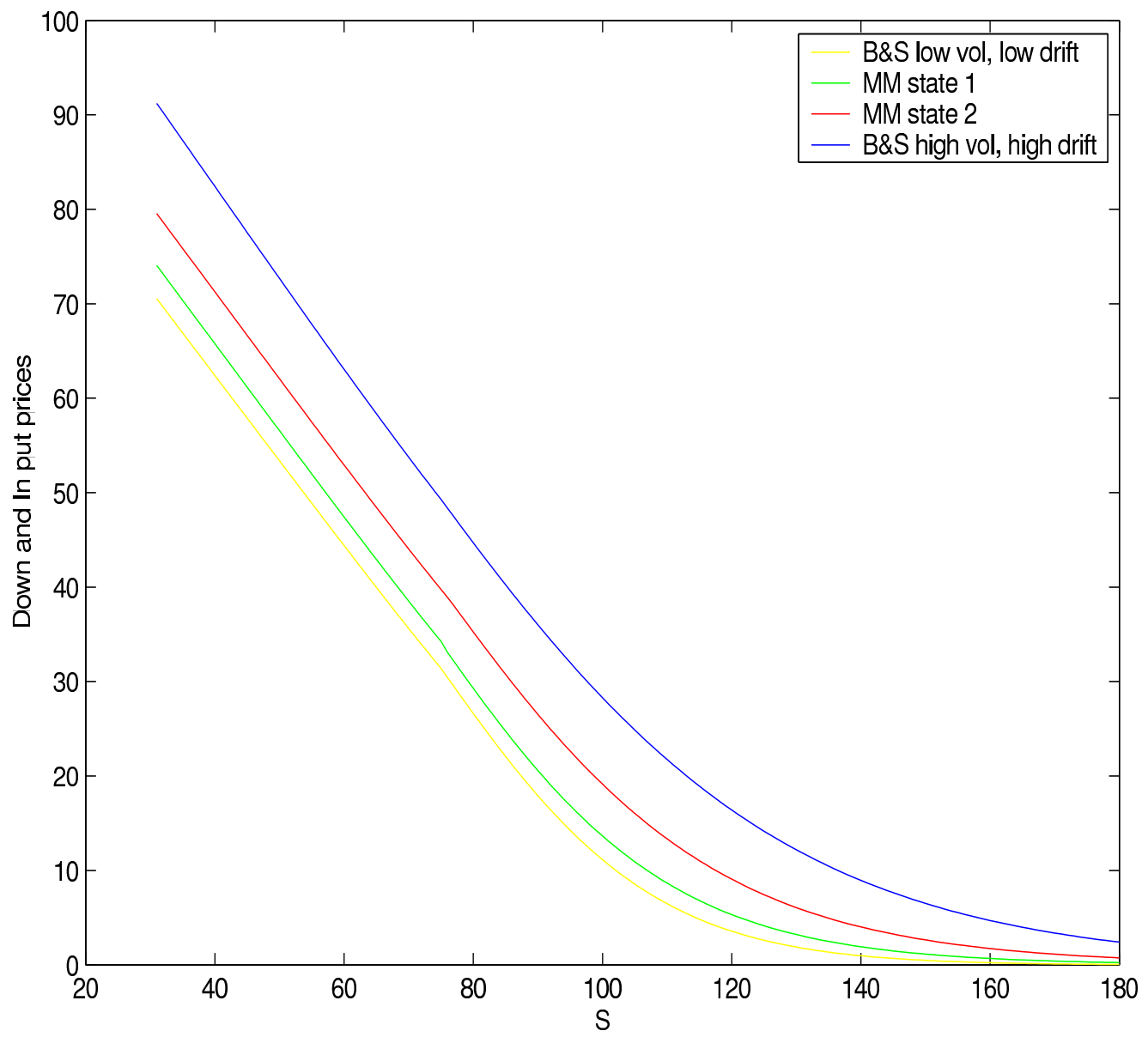

Figure 2: Comparison of put prices. 